\title{
A Rainbow of Colors and Spectrum of Textures: An Approach to Oral Mucosal Entities
}

\author{
Brenda L. Nelson ${ }^{1}$ - Lester D. R. Thompson ${ }^{2}$ (I)
}

Received: 20 December 2018 / Accepted: 2 January 2019 / Published online: 29 January 2019

○) Springer Science+Business Media, LLC, part of Springer Nature 2019

Nearly all surgical pathologists will encounter oral mucosal biopsies in their day to day practice. Many of these biopsies are fraught with diagnostic difficulty and may be challenging, usually due to inadequate clinical information, an unfamiliarity with anatomic landmarks and/or terminology used by the submitting physician or dentist, and the often limited size of the sample. A clinical description and history to accompany the submitted biopsy is often essential to the proper diagnosis and subsequent treatment of many oral conditions. A clinical history is a requirement recognized by the College of American Pathologist (CAP) [1]. The value and utility of a clinical history in the practice of pathology is undisputed. However, between 5.9 and $8.4 \%$ of cases submitted for pathologic review have no clinical history [1], or include vague information like "lesion," "bump" or "ulcer." Often the exact anatomic site is not included, stating only "oral cavity," or "soft tissue" without any further designation as to exact anatomic site. This lack of important information may result in less than ideal interpretations. Additionally, concise clinical histories have been associated with shorter sign-out turnaround times [2]. Pathologists find it too difficult to communicate with providers to obtain additional clinical information, claiming they are not in the office, they don't return messages, or clinicians are too busy to be bothered. With nearly ubiquitous access to photography, the old adage, "A picture is worth a thousand words," would seem to go a long way towards providing further clinical information. The clinical appearance, with the various nuances of color, texture and location are well suited to clinical photography. Cerroni et al., studied the utility of submitting clinical

Lester D. R. Thompson

brenda.1.nelson24.mil@mail.mil

1 Department of Anatomic Pathology, Naval Medical Center San Diego, 34800 Bob Wilson Drive, San Diego, CA 92134-5000, USA

2 Department of Pathology, Southern California Permanente Medical Group, Woodland Hills, CA, USA photographs with biopsy requests to dermatopathologists [3]. The study showed that clinical photographs facilitated better pathology interpretation. Further, Ferrara et al., highlighted that clinical photographs do not introduce bias into the diagnosis of melanocytic lesion of the skin [4]. There is a long held misconception by many physicians and dentists that clinically describing the sampled lesion unduly biases the pathologist: how patently false. Clear and concise clinical descriptions of mucosal lesions are essential to developing an appropriate differential diagnosis and a subsequent accurate interpretation. In an effort to highlight the importance of these characteristics, the topics covered by the contributing authors to this special issue have been divided by color, texture and location (Fig. 1).

Dr. Susan Müller discusses the clinical and histologic features of reactive white lesions of the oral mucosa. While many publications focus on leukoplakia and other potentially malignant lesions, most white lesions of the oral cavity are common reactive conditions. Dr. Müller discussed frictional keratoses, irritant contact stomatitis, smokeless tobacco keratosis and their differential diagnoses.

Dr. John W. Hellstein explores candidiasis, a common red and white lesion of the oral cavity, with an innovative and unique clinical perspective. His review concentrates on intraoral and perioral manifestations of the disease and its treatment. Various forms of oral and maxillofacial candidiasis are reviewed to include pseudomembranous, acute, chronic, median rhomboid glossitis, perioral dermatitis and angular cheilitis.

Erythematous (red) lesions of the oral mucosa are reviewed by $D r$. Kristin K. McNamara. These conditions reflect a variety of processes and etiologies, ranging from benign reactive or immunologically-mediated disorders to malignant diseases. The assessment focuses on salient clinical features and histopathologic findings of selected conditions, including: oral vascular malformations and neoplasms, pyogenic granuloma, localized 


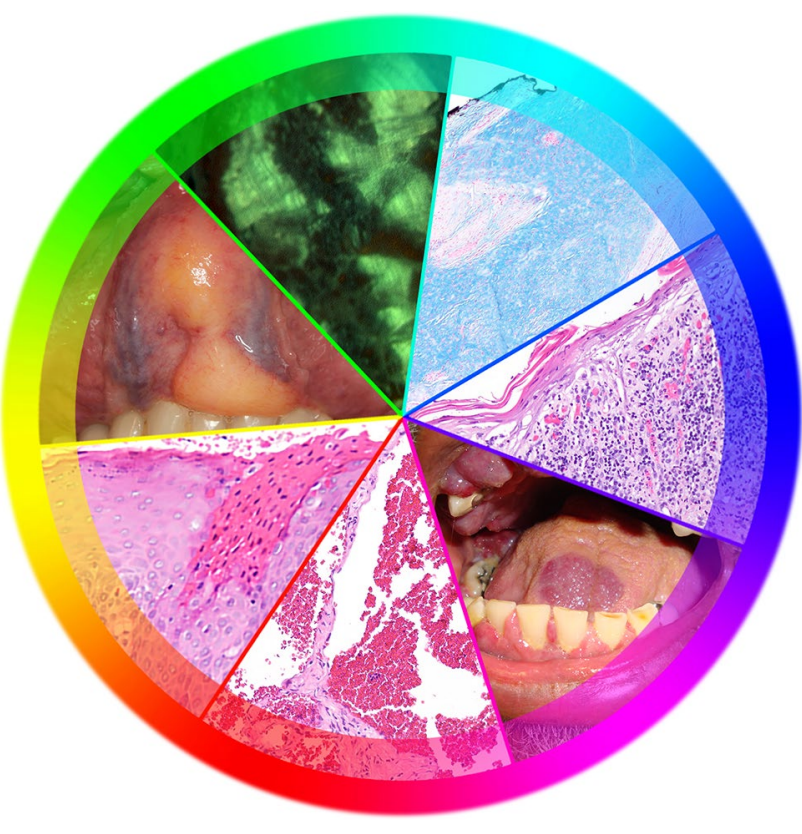

Fig. 1 This diagram highlights the many clinical colors of various lesions seen clinically. Each segment of this pie-chart highlight clinical or histologic features that match a specific color of the rainbow: Green (amyloid Congo Red birefringence), blue (Alcian blue myxoid stromal material), indigo (inflammatory infiltrate of lymphocytes), violet (a Kaposi sarcoma of the tongue), red (hemangioma), orange (keratosis and parakeratosis) and yellow (ventral tongue lipoma)

juvenile spongiotic gingival hyperplasia, denture stomatitis, and benign migratory glossitis (geographic tongue), among others.

Dr. Duane R. Schafer discusses the small but distinct group of yellow mucosal entities. Naturally occurring yellow components of the mucosa are very common, including adipose tissue, lymphoid tissue, and sebaceous glands. Additionally, reactive and inflammatory lesions may appear yellow due to purulence, necrosis, and calcification. Systemic diseases known to yield yellow mucosal lesions are detailed. Finally, while not always yellow, unusual entities like verruciform xanthoma and granular cell tumor are mentioned.

Pigmented brown and black lesions or the oral cavity with etiologies other than neoplasia are reviewed by $\mathrm{Dr}$. Molly S. Rosebush. Physiologic, reactive, and idiopathic melanin production seen in smoker's melanosis, drug-related discolorations, melanotic macule, melanoacanthoma and systemic diseases are covered, while including amalgam tattoo and black hairy tongue as exogenous sources of pigmentation. Key features highlight significant pigmented mucosal lesions, highlighting when to biopsy.

Dr. Easwar Natarajan presents the challenges around black and brown mucocutaneous neoplasms. His in depth approach will review the classic clinical and microscopic features of head and neck nevi, in addition to discussing emerging concepts in nevus pathogenesis and taxonomy.
Melanoma is discussed in detail with a focus on etiology, clinical presentation and histologic features.

Dr. Sarah G. Fitzpatrick considers ulcerated lesions of the oral cavity and their many underlying etiologic factors: infection, immune related, traumatic, or neoplastic. She stresses the need for a detailed patient history when assessing such lesions to include a complete medical and medication history; whether an inciting or triggering trauma, condition, or medication can be identified; the length of time the lesion has been present; the frequency of episodes in recurrent cases; the presence or absence of pain; and the arc of development over time. A clinical impression can be confirmed by biopsy, with certain lesions lending themselves to biopsy interpretation. The review includes a discussion of adjunctive and laboratory tests indicated to determine an autoimmune or systemic etiology.

Dr. Gisèle N. Mainville details oral mucosal papillary lesions of a reactive, neoplastic and non-HPV etiology. Her review provides coverage of the histopathological features of the most commonly encountered conditions: normal anatomic structures (retrocuspid papillae, lingual tonsils), reactive lesions (hairy tongue, inflammatory papillary hyperplasia), neoplastic lesions (giant cell fibroma), and lesions of unknown pathogenesis. Other papillary lesions associated with syndromes or representing paraneoplastic conditions are briefly presented. Common questions regarding differential diagnosis, management, and diagnostic pitfalls are addressed, stressing the importance of clinicopathologic correlation and collaboration.

HPV associated proliferations (squamous papilloma, condyloma acuminatum, verruca vulgaris, multifocal epithelial hyperplasia) are discussed by Dr. Sasha J. Betz. Mechanisms of infection, epidemiology, clinical presentations, histologic features, and differential diagnoses of the HPV-related oral pathologies are explored. Current concepts of viral transmission, especially as pertaining to lesions in pediatric patients, and the impact of HPV vaccination are reviewed.

Tongue exophytic lesions are discussed by Dr. Irit Allon. This diverse spectrum of reactive and neoplastic, lumps and bumps of the tongue are presented with clues to assist in reaching an accurate diagnosis, set within the context of clinicopathological correlation.

Finally, Dr. Keith D. Hunter emphasizes diagnosis of gingival lumps and bumps. Common lesions are covered, many of which are reactive hyperplasias, related to a chronic irritant stimuli, along with less common lesions. The clinical and histological differential diagnoses are covered to expose potential pitfalls.

In summary, these reviews of oral mucosal entities which emphasize the clinical appearance, including color, texture and anatomic location, should assist practicing pathologists to render a more complete and meaningful diagnosis. These conditions of the oral mucosa really do represent a 
rainbow spectrum, intended to stimulate further discussion and understanding of these diverse conditions.

\section{Compliance with Ethical Standards}

Conflict of interest The authors have no conflict of interest.

Ethical Approval This article does not contain any studies with human participants or animals, performed by either of the authors.

Disclaimer The opinions and assertions expressed herein are those of the author and are not to be construed as official or representing the views of the Department of the Navy or the Department of Defense or Southern California Permanente Medical Group.

\section{References}

1. Layfield LJ, Factor RE, Jarboe EA. Clinician compliance with laboratory regulations requiring submission of relevant clinical data: a one year retrospective analysis. Pathol Res Pract. 2012;208(11):668-71.

2. Ali SMH, Kathia UM, Gondal MUM, Zil-E-Ali A, Khan H, Riaz S. Impact of clinical information on the turnaround time in surgical histopathology: a retrospective study. Cureus. 2018;10(5):e2596.

3. Cerroni L, Argenyi Z, Cerio R, et al. Influence of evaluation of clinical pictures on the histopathologic diagnosis of inflammatory skin disorders. J Am Acad Dermatol. 2010;63(4):647-52.

4. Ferrara G, Annessi G, Argenyi Z, et al. Prior knowledge of the clinical picture does not introduce bias in the histopathologic diagnosis of melanocytic skin lesions. J Cutan Pathol. 2015;42(12):953-8. 